\title{
Using Signal Phase in Computerized Systems of Non-destructive Testing
}

\author{
Zhong Mei $^{1}$, Yurii Kuts ${ }^{2}$, Orest Kochan ${ }^{3,4}$, Iuliia Lysenko ${ }^{2}$, Oleksandr Levchenko², \\ Halyna Vlakh-Vyhrynovska ${ }^{4}$ \\ ${ }^{1}$ School of electronics \& information Engineering ( School of big data science), Taizhou university, Taizhou 318000, China, \\ zhongmei160620@gmail.com. \\ ${ }^{2}$ Igor Sikorsky Kyiv Polytechnic Institute, Kyiv, Ukraine, y.kuts@ukr.net. \\ ${ }^{3}$ School of Computer Science, Hubei University of Technology, Wuhan 430068, China; \\ ${ }^{4}$ Lviv Polytechnic National University, Lviv, Ukraine orest.v.kochan@lpnu.ua, halyna.i.vlakh-vyhrynovska@lpnu.ua
}

\begin{abstract}
Phase methods of measuring physical quantities and phase measuring equipment are widely used in various fields of science and technology. The article proposes a signal processing methodology based on a combination of the discrete Hilbert transform (DHT) and deterministic, as well as statistical methods of phase measurement. This methodology makes it possible to more fully use the information resource of the measuring signal phase in a wide range of the signal-to-noise ratio. It can be used both in computerized measurement and testing systems, as well as in the processing of measurement data. The benefits of the DHT are considered. The possibility of using statistics of directional data for phase measurements is shown. Circular statistics, such as the mean ring value, circular variance and the resulting vector length, were proposed for use in the phase measurements. Some examples of the use of this methodology in measurements and non-destructive testing are given.
\end{abstract}

Keywords: signal processing, phase measurement, discrete Hilbert transform, circular statistics.

\section{INTRODUCTION}

This paper is an extended and updated version of [1]. It is known that the measuring technologies are penetrating deeper into various areas of human activity because no science is possible without measurements [2]. In particular, the development of the concept of the internet of things [3], together with the progress in measurement science led to the exponential growth of measurement data. The rise of the computational power of computers created the opportunity to develop methods for measurement data processing to improve the accuracy of measurement and their noise immunity. This trend is illustrated by research in various areas of measurements and testing: measurement of highfrequency electromagnetic fields, which can have a negative impact on both the human body and electronic devices [4], precision temperature measurements [5], using fractal dimension for investigating surface parameters for fracture analysis after bending-torsion fatigue [6].

In some branches of science and technology, the current trend is the creation of measuring instruments and selfvalidating sensors [7] and in situ measurements [8], due to which there appear appropriate methods: the analysis and classification method to evaluate the working condition of angle grinders by means of infrared thermography and image processing [9], the method of thermocouple selfverification on operation place [10] and its application [11]. Some methods were also developed in other fields of science and technology, even without any program documents from metrological organizations: the method of assessment of the stress-strain-time and strength characteristics of geomaterials [12], the new strategy of the reliability monitoring of sensor arrays [13], the method of designing heat exchangers, which are used to heat or cool the material streams [14].

Development of computers also led to considerable progress in statistics and data processing algorithms [15] (the problems in modeling sensor degradation is considered and a new method of data fitting is proposed), [16] (the method of correlation analysis of natural water quality is investigated), including the methods of data science [17] (the use of neural networks to correct and compensate for sensor errors is proposed), [18] (the fault diagnosis method based on the analysis of thermal images is described).

Among the important areas of measurement science is the measurement of phase differences of harmonic and

DOI: $10.2478 / \mathrm{msr}-2022-0004$ 
quasiharmonic signals, which might be very suitable for developing the methods of self-validation and in situ measurements. This paper is devoted to the development of such a technique in phase methods of measuring physical quantities.

Using the signal phase as an information parameter allows effectively solving many problems in diverse fields of science and technology, e.g., in experimental physics, radiophysics, radio and radar navigation, telecommunications, geodesy, non-destructive testing, etc. The expansion of the signal phase application required the improvement of both deterministic [19] and statistical [20] methods for phase shift measuring. Based on the phase methods, many important scientific and technical problems related to the accurate measurement of distances, time intervals, azimuth, analysis of the characteristics of signal fields of different physical nature and others were solved.

The application of the phase methods required the creation of appropriate phase measuring equipment. At the initial stage of the development of the phase measuring equipment, the measurement conversion of signals' phase shifts to either the rotation angle of the electromechanical instrument's arm or constant voltage [21]. Another classic approach was based on the zero-crossing detection and conversion of the signals' phase difference to time intervals. At that time there had already existed the accurate voltage measures and time interval measures that allowed simplifying the phase measuring equipment. Then later the two-phase generators, which generated two coherent signals with a fixed phase shift between them, began to be used as a measure of signals' phase shift. This approach determined further development of the compensation method of phase measurements.

The current stage of development of phase measurements is associated with the use of digital signal processing [22]-[24]. The measurements of phase differences based on the discrete Fourier transform [24] and the method based on the sine-wave-fitting [25] are being actively improved. The methods of estimation of phase difference based on data extension and the Hilbert transform were presented in [22], [26]. In general, in recent decades there was an increase in the number of publications on phase measurements [27]-[31]. The latest studies showed the Hilbert transform can considerably improve noise immunity of measuring systems [22], [26].

In many technical applications, the result of phase measurements is stochastic. There are various reasons for this: unstable, probabilistic nature of the signal source; noise in the signal transmission channel; noise in phase measuring equipment, and so on. Therefore, there is a need for using statistical processing of phase data to improve the metrological characteristics of the phase measurement results. The methods of statistical analysis of random variables are well known. They are well studied and simple for hardware implementation which contributes to their wide use in the phase measurement techniques. However, the transfer of the methods of statistical analysis of random variables to the field of measurements of phase differences to process the signal has its limitations. This is because the phase of periodic signals has the natural periodicity of $2 \pi$, and thus for the phase difference $\varphi$ there is a mathematical operation $\varphi \equiv(\varphi+2 \pi k) \bmod 2 \pi, k=0, \pm 1, \pm 2, \ldots$. This feature should be taken into account when justifying the algorithms for processing phase data, and when forming the measurement result.

To obtain the measurement result as a set of quantity values being attributed to a measurand together with any other available relevant information [32], it is necessary to use two measures. The first is a material measure used to obtain the measurement quantity value. It is a measuring instrument reproducing or supplying quantities of one or more given kinds, each with an assigned quantity value. The second is a probability measure that is generated by a certain probability distribution. It is used to estimate the measurement uncertainty as a non-negative parameter characterizing the spread of quantity values being attributed to a measurand. To obtain the correct results of the phase measurements, it is necessary to adhere to the concept of harmonization of these measures [33] and to reconcile them with the circular nature of the phase as a measurand.

Nowadays, the achievements of computer technology and digital signal processing have created the preconditions for improving the methodology of phase measurement processing, implementation of complex algorithms for statistical processing of phase data, and more complete use of the information resource of the signal phase.

This article discusses the method of obtaining and analyzing the signal phase based on a combination of the discrete Hilbert transform (DHT), which allows obtaining a sample of signal phases, and their statistical processing as well as the ability of this method to more fully disclose the information resource of the signal phase. In addition, the article shows the analogy of the statistical analysis of angular and phase data, considers the calculations of the phase deterministic and stochastic characteristics, and gives examples of their application in various measurements and non-destructive testing.

\section{FoRMULATION OF THE PROBLEM}

A phase measurement experiment is performed. Both the measurement signal and the reference signal are represented by the expressions as follows

$$
\begin{aligned}
& u(t)=U(t) \cos [2 \pi f t+\varphi(t)]+n(t), \\
& u_{r}(t)=U_{r} \cos \left[2 \pi f t+\varphi_{r}\right], \quad t \in T_{o},
\end{aligned}
$$

where $U(t)$ and $\varphi(t)$ are an envelope and a phase of the measurement signal $u(t)$, respectively; $U_{r}$ and $\varphi_{r}$ are the amplitude and the initial phase of the reference signal $u_{r}(t)$, respectively; $t, T_{0}$ are time and time interval of signal observation, respectively; $f$ is the frequency of the harmonic signals; $n(t)$ is the implementation of white noise with zero expectation and $\sigma^{2}$ variance. The functions $U(t), \varphi(t)$ are slowly changing functions of $t$.

Fourier transform exists for signals (1). After digitization of these signals, they are represented by samples $\{u[j], j=\overline{1, J}\},\left\{u_{r}[j], j=\overline{1, J}\right\}, J=\operatorname{int}\left(T_{o} / T_{s}\right), T_{s}$ is 
a sampling interval. It is necessary to consider the use of the signal phase to calculate the derived characteristics and to confirm their efficiency for measurements and non-destructive testing.

The material of the article is presented in the following order: the DHT and its application for signal phase calculation; probabilistic space and model of random angles and random phase shift of signals; some circular statistics for the use in the phase measurement; probability distributions of random angles for the use in the phase measurements; application of the proposed methodology in some applied problems of phase measurements and non-destructive testing (NDT); conclusions.

\section{DiSCRETE HILBERT TRANSFORM AND ITS APPLICATION TO CALCULATE THE SIGNAL PHASE}

The DHT and the concept of "analytical signal" introduced on its basis are widely used for theoretical studies of periodic processes and phenomena [34], [35]. The discrete analytical sequence [36], [37] is determined as $\dot{z}[j]=u[j]+i \tilde{u}[j]$, where $\tilde{u}[j]$ is the DHT of $u[j]$ and $i=\sqrt{-1}$. The simplest method for calculating the DHT is the spectral method. It can be found in [38]. Using the definition of a discrete analytical sequence one can find the discrete instantaneous phase of the sequence $u[j]$

$$
\begin{aligned}
& \hat{\varphi}[j]=\tan ^{-1} \tilde{u}[j] / u[j]+ \\
& +0,5 \pi\{2-\operatorname{sign} \tilde{u}[j](1+\operatorname{sign} u[j])\} .
\end{aligned}
$$

Function (2) has a sawtooth shape and changes periodically within the range of $[0,2 \pi)$. Thus, it can be considered as a wrapped phase of a signal. The discrete instantaneous unwrapped phase can be obtained from (2)

$$
\hat{\Phi}[j]=\hat{\varphi}[j]+2 \pi g(\hat{\varphi}[j])
$$

where $g(\hat{\varphi}[j])$ is a step function, which increases by one each time the phase changes from $2 \pi$ to 0 . Equation (3) estimates the instantaneous unwrapped phase $(2 \pi f t+\varphi(t))$ of the signal $u(t)$ as a function of time. If necessary, the estimate of the unwrapped phase $\hat{\Phi}_{r}[j]$ of the signal $u_{r}[j]$ can also be obtained according to (3), having determined $\tilde{u}_{r}[j]$ as the DHT of $u_{r}[j]$ prior to this.

The discrete instantaneous phase shift is determined as follows

$$
\hat{\varphi}_{j}=\hat{\Phi}[j]-\hat{\Phi}_{r}[j]
$$

Equation (4) also allows calculating the phase difference if $\varphi(t)=$ const: $\hat{\varphi}_{j}=\varphi-\varphi_{r}$. When $\varphi_{r}=0$ and the signal frequency is known, then the discrete instantaneous phase shift can be obtained as follows

$$
\hat{\varphi}_{j}=\hat{\Phi}[j]-2 \pi f T_{s} j, j=\overline{1, J} .
$$

Using the unwrapped phase (3), one can get the instantaneous signal frequency according to the following formula

$$
\hat{f}[j]=\frac{(\hat{\varphi}[j]-\hat{\varphi}[j-1]) \bmod 2 \pi}{2 \pi T_{s}}, j=\overline{2, J}
$$

Thus, the use of the DHT allows one to simultaneously obtain the large samples of an instantaneous amplitude, phase, phase shifts, and frequency of the analyzed signal. This creates the preconditions for more correct use of statistical methods of their processing. Graphical representation of the algorithms for obtaining samples and calculating circular statistics for phase and angular measurements is given in Fig.1.a) and Fig.1.b).

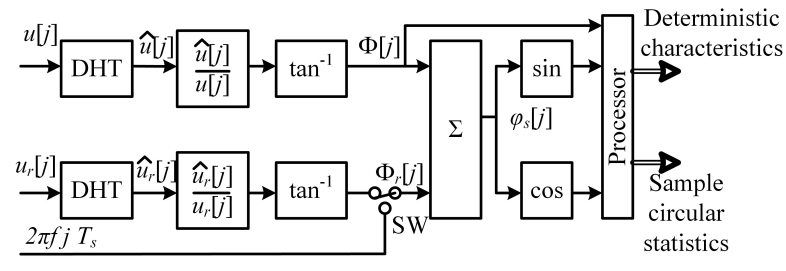

a)

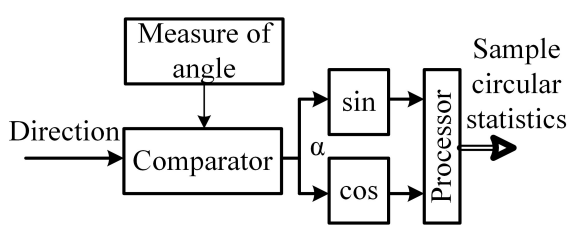

b)

Fig.1. Graphical representation of algorithms for calculating circular statistics for phase a) and angular b) measurements.

If in the angular measurements (Fig.1.b)) the quantity value of the angular is determined using the physical angle measure such as circular scales, limbs, etc., the DHT allows one to determine the quantity value of the signal phase without any physical measures (Fig.1.a)).

The DHT has other important properties suitable for phase measurements. These are as follows:

- due to the fact that the DHT has the property of linearity [34] and has the magnitude of the transfer function equal to unity, the signals $\tilde{u}[j], \tilde{u}_{r}[j]$ are determined without distortions of the measure of appropriate signals $u[j], u_{r}[j]$;

- the phase (2) and phase shift (3) measurements do not require the use of a physical measure of phase;

- due to the fact that $T_{s}<<1 / f$, it is possible to determine the phase changes of the signals on the time intervals significantly shorter than the signal period (4); 
- the ability to determine the phase of a phase-modulated signal or phase-shift keying signal (5);

- to use the phase and phase shifts of the signals to obtain some deterministic characteristics: the instantaneous wrapped phase and unwrapped phase of the signal (2), (3); the instantaneous phase shift of signals (4), (5); the instantaneous signal frequency (5);

- to use the large samples of phase difference (4) of significant volume $J$ to obtain circular statistics such as trigonometric moment, circular mean; length of the resultant vector, circular variance, etc. (these circular statistics will be discussed below).

The most important derived features of a signal phase are given in Fig.2.

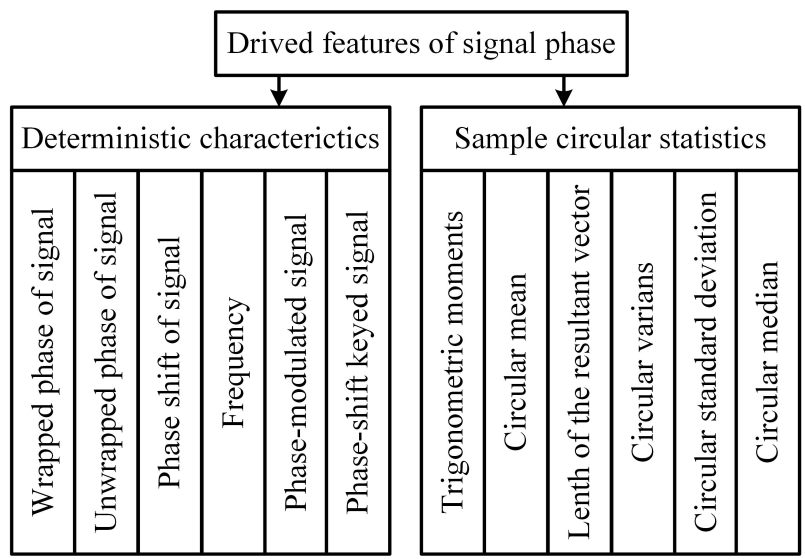

Fig.2. The classification of the derived features of the signal phase.

The deterministic characteristics include:

- the wrapped phase of the signal, it is determined in a discrete form according to (2);

- the unwrapped phase of the signal, it is determined in a discrete form according to (3);

- the phase shift of the signal, it is determined in a discrete form according to (4);

- the frequency (6);

- the phase-modulated signal and phase-shift keyed signal, they are determined in a discrete form according to (4), where $\hat{\Phi}_{r}[j]$ is the unwrapped phase of the unmodulated reference signal.

The sample circular statistics will be discussed in section 4 .

\section{PRobabilistic SPACE AND MODEL OF RANDOM ANGLES AND RANDOM PHASE DIFFERENCES}

In the case of estimating the parameters of harmonic signals, which are the components of the additive mixture with noise, the problem of processing random phase shifts occurs. Since the models of random angles are similar to the models of random phase shifts [33], the methods of statistical processing of angular data [36], [37] can be applied to them.

One of the main objects of statistical analysis of circular data is a vector $\overline{\boldsymbol{z}}$ that can have different directions on the
Q plane. Fig. 3a shows the set of vectors $\overline{\boldsymbol{z}}_{j}, j=\overline{1,7}$ as an example. Since the modulus of the vector is irrelevant for angular observations, the directions on the Q plane are given by vectors of unit length. The direction of vectors $\overline{\boldsymbol{z}}_{j}, j=\overline{1, J}$ is considered in the theory of probabilities of random angles as an elementary random event $\omega_{j}$. The probabilistic content of the experiment with unit vectors will not change if we transfer the beginning of all vectors to point $\mathrm{O}$ on the $\mathrm{Q}$ plane and consider the corresponding set of collinear vectors (Fig.3.b)).

The set of all possible events $\omega_{j}, j=\overline{1, J}$ forms a sample space $\Omega=\left\{\omega_{1}, \ldots \omega_{j}, \ldots \omega_{J},\right\}$, which is reflected by the points on a unit circle (Fig.3.b)).

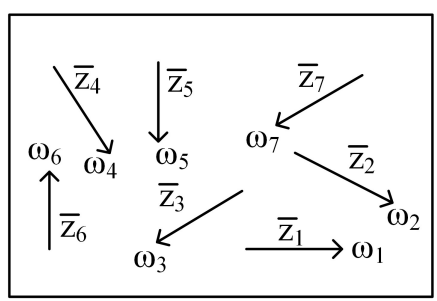

a)

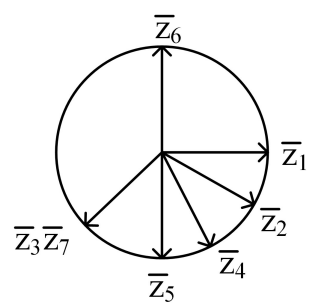

b)
Fig.3. Display of the elementary events on the plane $Q$ a) and on the unit circle b).

In the space $\Omega$, we set a random angle as a numerical function $\psi(\omega) \in[0,2 \pi)$. To do this we use the cylindrical coordinates [33]. In the general case, the set of elements in space $\Omega$ has the power of a continuum. The infinite number of directions of the vector $\overline{\boldsymbol{z}}$ significantly complicates the presentation of the experimental results.

It is more practical to divide the space of directions on the plane into a finite set of $n$ events, which correspond to certain sectors. All vectors within each sector are considered to correspond to one elementary event (Fig.4.).

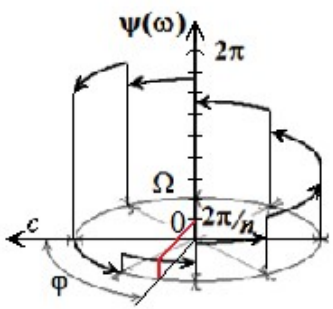

Fig.4. Determination of a random angle.

For a random phase difference, one can formally use the same probability space and model. The difference is that the input value in this model is the phase difference.

The same probabilistic space in the form of a unit circle has a random phase difference. Therefore, the methods of angular data analysis can be applied to it and the sample 
circular characteristics can be determined. The most important sample circular characteristics in phase measurements are discussed below.

\section{QUANTITATIVE CHARACTERISTICS OF THE RANDOM PHASE DIFFERENCE ESTIMATED FROM THE SAMPLE}

According to the results of measurements, the sample of phase differences $\left(\varphi_{1}, \ldots \varphi_{j}, \ldots \varphi_{M}\right), \varphi_{j} \in[0,2 \pi)$, of size $\mathrm{M}$ is obtained. This sample is considered as the realization of random angles having a certain continuous probability density function. The quantitative characteristics of the sample of the phase differences are determined as follows.

$A$. The sample trigonometric moment of order $n$ relative to a given phase difference $\alpha \in[0,2 \pi)$ is determined by the formula as follows:

$$
\begin{aligned}
& \hat{f}_{n}(\alpha)=M^{-1} \sum_{j=1}^{M} e^{i u\left(\phi_{j}-\alpha\right)}= \\
& =\hat{a}_{n}(\alpha)+i \hat{b}_{n}(\alpha)=\hat{r}_{n}(\alpha) e^{i \hat{m}_{n}(\alpha)}
\end{aligned}
$$

and the sample cosine and sine moments of order $n$ are calculated according to the formulas:

$$
\begin{aligned}
& \hat{a}_{n}(\alpha)=M^{-1} \sum_{j=1}^{M} \cos \left[n\left(\varphi_{j}-\alpha\right)\right], \\
& \hat{b}_{u}(\alpha)=M^{-1} \sum_{j=1}^{M} \sin \left[n\left(\varphi_{j}-\alpha\right)\right] .
\end{aligned}
$$

Sample characteristic function is a complex-valued sequence $\left(\hat{f}_{n}(0), n=0, \pm 1, \pm 2, \ldots\right), \quad$ all of whose trigonometric moments of the sample are determined with respect to the zero direction $\alpha=0$.

$B$. Sample circular mean is calculated by the formula

$$
\varphi_{\mathrm{c}}=\operatorname{arctg} S / C+0.5 \pi\{2-(\operatorname{sign} S)(1+\operatorname{sign} C)\}
$$

where

$$
C=M^{-1} \sum_{j=1}^{M} \cos \varphi_{j}, \quad S=M^{-1} \sum_{j=1}^{M} \sin \varphi_{j} .
$$

The result of a separate phase measurement $\varphi_{j}$ can be represented by the corresponding unit vector $\overrightarrow{O P_{j}}=\exp \left(i \varphi_{j}\right)$ in the $\mathrm{XY}$ Cartesian coordinate system as illustrated in Fig.5. The vector $\overrightarrow{O P_{j}}$ has Cartesian $\left[\cos \varphi_{j}, \sin \varphi_{j}\right]$ and polar $\left(1, \varphi_{j}\right)$ coordinates, respectively.

The sample circular mean $\varphi_{\mathrm{c}}$ has the following mechanical interpretation. If the same "mass" $M^{-1}$ is attributed to all points $\left[\cos \varphi_{j}, \sin \varphi_{j}\right]$, then the point $\left[\cos \varphi_{c}, \sin \varphi_{c}\right]$ will be the "center of mass" of this system.

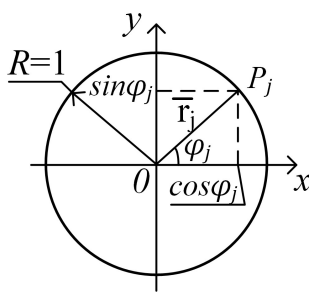

Fig.5. Display of the phase difference $\varphi_{j}$ on the unit circle.

C. Mean resultant length of the vector is calculated according to the formula

$$
r=\sqrt{C^{2}+S^{2}}
$$

D. Sample circular variance

$$
V=1-r
$$

E. Sample circular standard deviation

$$
\sigma=\sqrt{-2 \ln (1-V)}=\sqrt{-2 \ln r} .
$$

F. Sample median phase difference (direction). It is the phase difference which corresponds to the point of the circle $P$ such that the diameter $P Q$ divides the statistic value into two halves, and in the vicinity of $P$ there is a maximum concentration of values.

G. Sample asymmetry

$$
g_{1}=\frac{b_{2}(m)}{V^{3 / 2}}=\frac{r_{2} \sin \left[m_{2}(0)-2 m\right]}{V^{3 / 2}} .
$$

\section{J. Sample kurtosis}

$$
g_{2}=\frac{a_{2}(m)-(1-V)^{4}}{V^{2}}=\frac{r_{2} \cos m_{2}(0)-2 n-(1-V)^{4}}{V^{2}} .
$$

\section{PROBABILITY DISTRIBUTIONS OF RANDOM ANGLES FOR THE USE IN PHASE MEASUREMENTS}

Any measurement result consists of one measured value and an expanded measurement uncertainty with a stated coverage probability [32]. The coverage probability, as well as the coverage factor and coverage interval, can be evaluated from probability density functions. The probability distribution functions of random phase differences are the same as those of random angles. One of the characteristic features of the circle as a space, on which the probability distributions are formed, is the property of 
periodicity (with the period of $2 \pi$ ) of the laws of the probability density distribution of random phase difference. As a rule, the density distributions of random phase differences are considered within the interval $[0,2 \pi)$.

The von Mises probability distribution [36], [37] is determined by the formula:

$$
p_{M}(\varphi \mid \mu, k)=\exp \{k \cos (\varphi-\mu)\} / 2 \pi I_{0}(k) \text {, }
$$

where $I_{0}$ is the modified Bessel function of the first kind and zeroth order; $\mu$ is the circular mean direction of a random angle, $|\mu|<\infty ; k$ is the concentration parameter of a random angle in the vicinity of $\mu, k>0$.

The plots of the functions $p_{M}(\varphi)$ for various parameter values are shown in Fig.6.

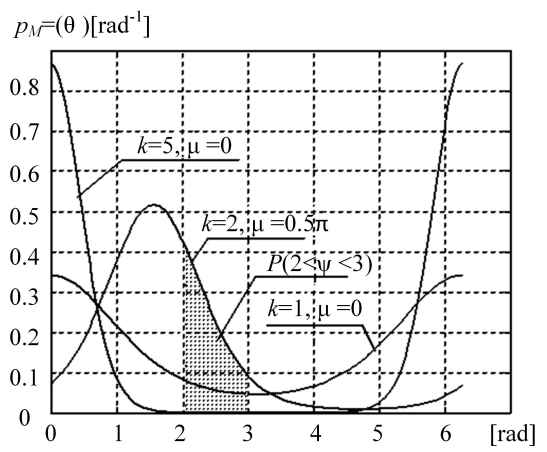

Fig.6. Examples of von Mises probability density functions.

This distribution is one-vertex and symmetric with respect to the mean value of $\mu$, which is the mathematical expectation of this distribution.

When increasing the parameter $\mathrm{k}$, the von Mises distribution concentrates around $\mu$ (if $\mathrm{k}=2$ and $\mu=0.5 \pi$, the distribution is almost completely concentrated on the arc from 0 to $3 \mathrm{rad}$, and if $k \rightarrow 0$ - converges to the uniform one).

The wrapped Normal distribution [36], [37] refers to the family of wrapped distributions ("wrapped" on a unit circle) formed by the nonlinear transformation of a random variable $\xi(\omega)$ into a random angle $\psi(\omega)$ of the form $\psi(\omega)=[K \xi(\omega)] \bmod 2 \pi$, where $K$ is the scale conversion coefficient. This transformation leads to the transformation of the laws of distribution on a straight line into the laws of probability wrapped distribution of random angles. If $\xi(\omega)$ has a probability distribution density $p(x)$, then a continuous random angle $\psi(\omega)$ also distributed continuously with the density is given as follows:

$$
p_{2 \pi}(\varphi)=\sum_{j=-\infty}^{\infty} p(\varphi+2 \pi j)
$$

In general, the function $p_{2 \pi}(\theta)$ is asymmetric with respect to the middle of the interval $[0,2 \pi)$, and at its ends acquires the same values: $p_{v}(0)=\lim _{\varphi \rightarrow 2 \pi} p_{v}(\varphi)$.

The density of the wrapped normal distribution is given as follows

$$
\begin{aligned}
& p_{2 \pi}(\varphi / \mu, \sigma)=\frac{1}{\sqrt{2 \pi} \sigma} \times \\
& \times \sum_{j=-\infty}^{\infty} \exp \left(-\frac{[(\varphi-\mu)(\bmod 2 \pi)+2 \pi j]^{2}}{2 \sigma^{2}}\right)
\end{aligned}
$$

where $\mu$ is the mathematical expectation; $\sigma$ is the standard deviation of the random variable $\xi(\omega)$. This law has the important property: the sum of the independent angles $\sum_{i=1}^{n} \varphi_{i}$, each of which has a distribution (19), also has the same distribution, but with different characteristics. In addition to the wrapped normal distribution, the central limit theorem holds on the circle: for independent random angles, which have the same probability distribution function, the probability distribution of the normalized sum of angles approaches to the wrapped normal distribution.

Examples of probability density plots of the wrapped Normal distribution for various values of the parameters are shown in Fig.7.a) and Fig.7.b), From these plots it can be seen that the wrapped normal distribution is one-vertex and symmetrical with respect to $\varphi=\mu(\bmod 2 \pi)$.
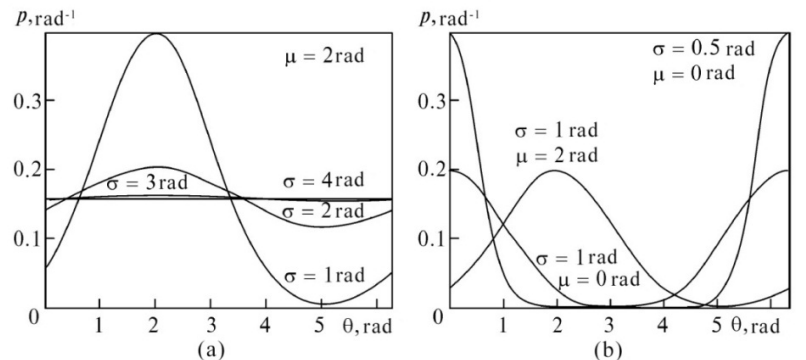

Fig.7. Density of the wrapped Normal probability distribution of random angles with various parameters.

If $\sigma \rightarrow \infty$ distribution converges to the uniform distribution with the density function of $1 / 2 \pi$, an increase of $\mu$ leads to a shift in the maximum of the function towards big angles. Within the interval of $[0,2 \pi)$, distribution (22) has two inflection points.

An appropriate choice of the parameters of the wrapped normal distribution allows giving a reasonable approximation for it by the von Mises distribution

Some other typical circle distributions, such as the uniform, triangular are presented in [36], [37]. 
The use of these distributions allows more correctly determining the standard uncertainty, coverage factor, coverage interval and coverage probability, which are necessary to estimate the accuracy of the phase measurements.

\section{APPLICATION EXAMPLES}

The DHT method is suitable for many applications, including NDT. In particular, the application of the proposed method of signal processing for pulsed eddy current test (PECT) problems is considered below.

\section{A. Application of phase signal characteristics for evaluating the diameter}

The experiments were performed with the testing object (TO) in the form of cylindrical samples and with the eddy current probe (ECP). Two sets of samples of size 11 were made of aluminum and bronze (specific conductivities $\gamma_{a l}=4.87 \cdot 10^{7} \mathrm{Sm} / \mathrm{m}, \quad$ and $\quad \gamma_{b r}=2.75 \cdot 10^{7} \mathrm{Sm} / \mathrm{m}$, respectively, with the diameter from 34 to $35 \mathrm{~mm}$ with a step of $0.1 \mathrm{~mm}$ ). The experiment was to determine the diameter of the TO using the value of frequency of natural oscillations of the ECP signal. This signal was obtained as a TO response to the pulse electromagnetic excitation field [39], [40].

The ECP signal is shown in Fig.8. It has the form of damped harmonic oscillations. The frequency and attenuation of the oscillations have been determined by the phase and envelope of the probe signals, respectively. The probe signal phase from the sample with $\mathrm{D}=34 \mathrm{~mm}$ is shown in Fig.9.

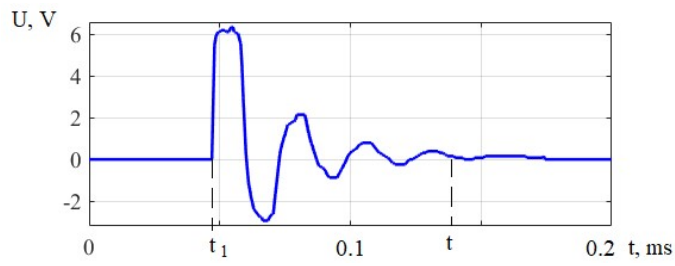

Fig.8. The plot of the ECP signal.

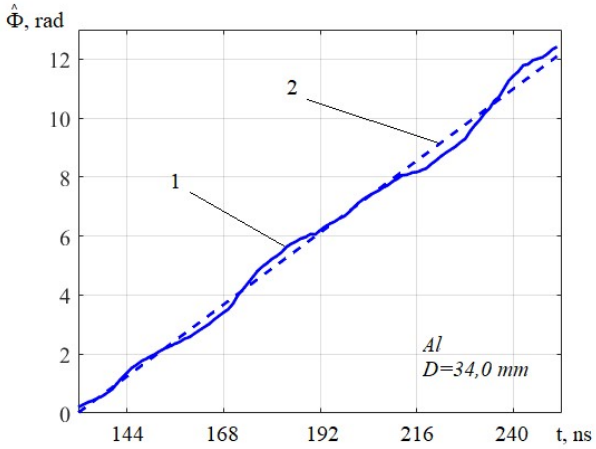

Fig.9. Examples of functions $\widehat{\Phi}(t)(1)$ and $\widehat{\Phi}_{L}(t)(2), D=34 \mathrm{~mm}$.
It is considered that the function $\widehat{\Phi}(t)$ has a linear nature and the frequency determination was performed using its linear trend $\widehat{\Phi}_{L}(t)[41]$ :

$$
f_{L}(\Delta T, D)=\frac{\Delta \widehat{\Phi}_{L}(\Delta T, D)}{2 \pi \Delta T}
$$

where $\Delta \widehat{\Phi}_{L}(\Delta T, D)$ is the accumulated phase of the ECP signal during the time interval $\Delta T=t_{2}-t_{1}$. The function $\widehat{\Phi}_{L}(t)$ can be obtained, e.g., by the Bartlett-Cenoa method. Examples of functions $\widehat{\Phi}_{L}(t)$ for aluminum specimens with the diameters of $34.7 \mathrm{~mm}$ and $34.0 \mathrm{~mm}$ are given in Fig.10. as curves 1 and 2, respectively.

The plot of the function for sets of aluminum (curve 1) and bronze (curve 2) samples is presented in Fig.11. Comparative analysis of the obtained data shows that it is possible to determine the diameter of the samples by the proposed method with the absolute error of $0.1 \mathrm{~mm}$ (the relative error of $0.3 \%$ )

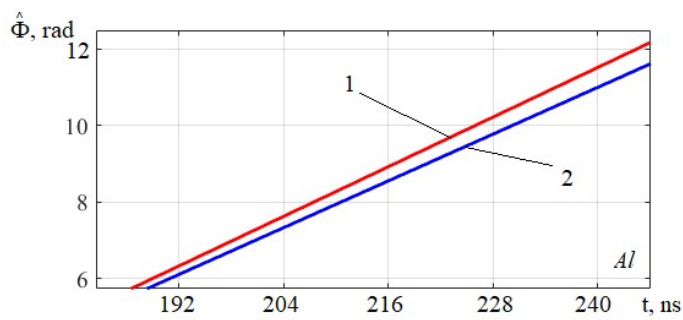

Fig.10. Examples of functions $\widehat{\Phi}_{L}[t]$ for aluminum specimens with the diameters of $34.7 \mathrm{~mm}$ (1) and $34.0 \mathrm{~mm}$ (2), respectively.

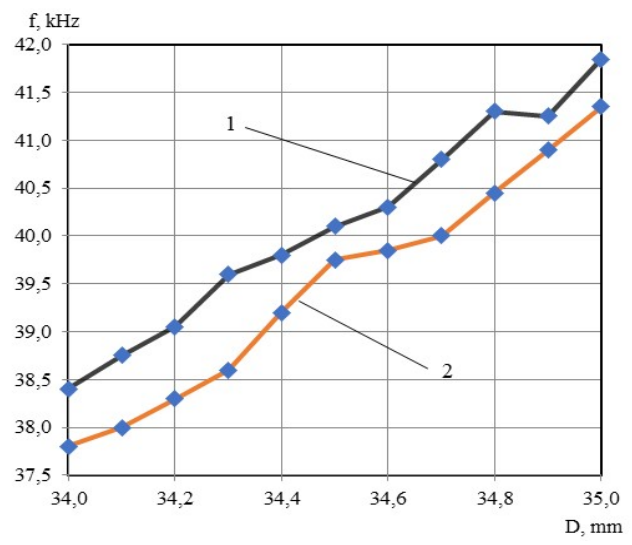

Fig.11. Functions of $f(D)$ : for aluminum a) and bronze b) TO.

The application of statistical processing methods to signal phase characteristics in the case of pulsed excitation allows decreasing the uncertainty of measuring the diameter of an object by more than 5 times in comparison with the case of ECP excitation by a harmonic signal. 
B. Determining a crack using the phase signal characteristics

The aluminum plate with cracks of various depths (from $0.1 \mathrm{~mm}$ to $3 \mathrm{~mm}$ ) with the width of $1 \mathrm{~mm}$ was used as the test specimen (TS) (Fig.12.). In order to determine the influence of TS parameters on the natural frequency of the probe signal, the scanning of the TS was performed with a step of $1 \mathrm{~mm}$ along the object length using a multidifferential probe [42], [43].

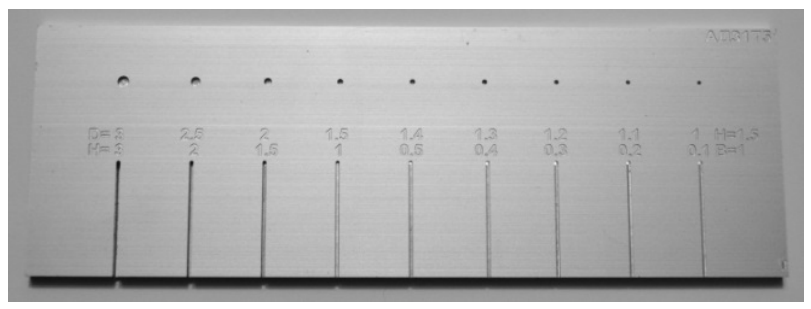

Fig.12. The test specimen.

According to the results of the TS scan, the set of probe signals was obtained, from which the value of frequency was selected with respect to coordinates.

The distribution of the frequency of ECP natural oscillations near the coordinates of cracks with the depths of $3,2,1.5$, and $1 \mathrm{~mm}$ (the coordinates of $14,32,50,68 \mathrm{~mm}$, respectively) is illustrated in Fig.13.

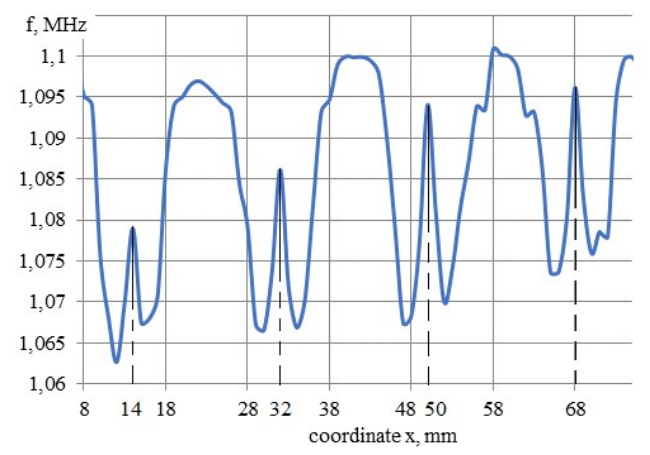

Fig.13. Dependence of ECP signal frequency on the coordinate.

The dependence in Fig.13. shows that in the locations of cracks there is a drop of the frequency of ECP natural oscillations near the crack and a rise directly above it.

Using the frequency of natural oscillations of the ECP signal as an informative parameter allows increasing the probability of determining a crack by approximately 10 $15 \%$.

\section{The evaluation of a phase difference measurement result}

The problem is formulated as follows [20]. The random phase difference $\psi(\omega) \in[0,2 \pi)$ of two signals is measured. It has the wrapped normal distribution (22). The measurement is performed under constant conditions. The sample $\left(\varphi_{1}, \ldots \varphi_{j}, \ldots \varphi_{M}\right), \varphi_{j} \in[0,2 \pi)$ is obtained, where $M$ is the sample size. It is considered as a realization of the random vector $\left(\psi_{1}, \ldots \psi_{j}, \ldots \psi_{M}\right)$ with independent random components that have the same distribution as $\psi(\omega)$. It is necessary to determine the measurement result.

The algorithm for determining the measurement result includes the following steps:

1. calculation of the sums $C$ and $S(11)$;

2. calculation of the sample circular mean $\varphi_{c}(10)$;

3. verification if the sample circular mean is correctly determined using the condition as follows [36]

$$
\sum_{j=1}^{M} \sin \left(\varphi_{j}-\varphi_{\mathrm{c}}\right)=0
$$

4. calculation of the sample circular variance $V(13)$;

5. calculation of the sample circular standard deviation $\sigma(14)$;

6. calculation of the standard uncertainty $u_{\varphi_{c}}$ of the sample mean

$$
u_{\varphi_{\mathrm{c}}}=\sigma / \sqrt{M}
$$

7. justification of the coverage probability $P_{c o v}$;

8. determination of the coverage factor $k[20]$;

9. calculation of the expanded uncertainty for the phase measurement: $U_{\text {cov }}=k u_{\varphi_{\mathrm{c}}}$;

10. calculation of the coverage interval for the coverage probability $\boldsymbol{P}_{\text {cov }}$

$$
\left(\varphi_{\mathrm{c}} \pm U_{\mathrm{cov}}\right) \bmod 2 \pi
$$

Determination of the coverage interval and the corresponding coverage probability for the circular data is shown in Fig. 14.

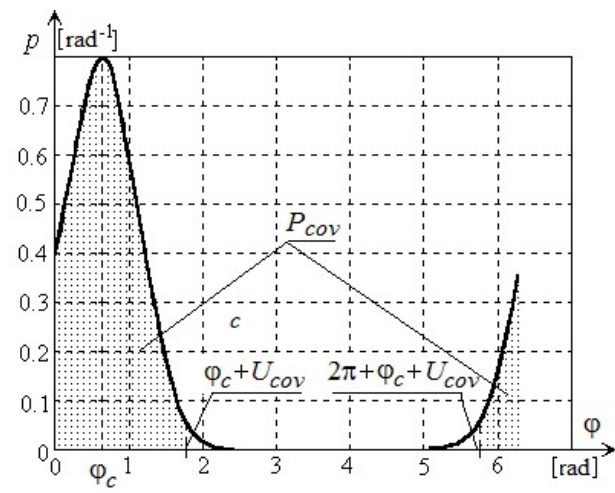

Fig.14. Graphical representation of the coverage interval and coverage probability in the case of circular data processing. 
Example. Let us measure a random phase shift that has the wrapped Normal distribution. According to the sample volume $M=100$, the sums are determined: $C=-0.0879$, $S=0.5964$.It is necessary to calculate the coverage interval for the coverage probability of 0.95 .

According to the above algorithm we have the following results:

$$
\begin{aligned}
& \varphi_{\mathrm{c}}=-\operatorname{arctg} \frac{0.5964}{0.0879}+\frac{\pi}{2}\{2-(1)(1+(-1))\}=1.717 \mathrm{rad}, \\
& V=1-\sqrt{(-0.0879)^{2}+0.5964^{2}}=0.3972, \\
& \sigma=\sqrt{-2 \ln (1-V)} \approx \sqrt{-2 \ln 0.6028} \approx 1.006, \\
& u_{\varphi_{\mathrm{c}}}=\sigma / \sqrt{M}=1.006 / \sqrt{100}=0.1006 \mathrm{rad} .
\end{aligned}
$$

In the case of $M=100$, the distribution of the averaged phase shift can be approximated by the Gaussian distribution. As $P_{\text {cov }}=0.95$, the coverage factor $k$ is equal to 2 , then

$$
U_{\text {cov }}=k u_{\varphi_{\mathrm{c}}}=2 \cdot 0,1006 \approx 0.20 \mathrm{rad} .
$$

Answer: the coverage interval for the coverage probability of 0.95 is equal: $(1.72 \pm 0.20)$ rad.

The proposed method evaluation of a phase difference measurement result assumes the use of several uncorrelated results of measuring the phase shift during one signal period, in contrast to traditional evaluation methods, which are focused on measuring one phase result per period. Thus, the described method improves the uncertainty by 4-5 times.

\section{Application of the mean resultant length of the vector for pulsed echo-method of ultrasonic measurements of the thickness}

This method is based on the determination of the time delay $\tau$ required for the propagation of the ultrasonic signal inside the testing object [44]. The thickness of the testing object can be determined as $h=0.5 \cdot c \tau$. It is assumed that the propagation velocity $c$ of the ultrasonic wave in two directions between the surface and the bottom of the object is known. The value of $\tau$ is usually estimated by the time interval between the envelopes of the two back wall ultrasonic signals. This method is very noise sensitive.

The general signal model of such measurements is represented by the top formula in (1). It is assumed that $U(\mathrm{t})>0$ for a limited time interval covers one or some more periods of the signal (1).

In case of structural materials testing with significant ultrasonic attenuation, there is a problem of detecting noisy ultrasonic pulse signals. This problem is effectively solved by using the mean resultant vector length for time interval measuring.

The proposed method of thickness measurement is based on such operations: forming an ultrasonic initial pulse, applying it to the TO, measuring the signal after its propagation through the object and reflection from the opposite side (back wall), determining the unwrapped phase of the echo-signals (3)), calculating the phase difference between the echoes and initial signals (4), processing the signals using the sliding window (with $M_{w}$ sample size), and calculating the lengths of the resultant vector (12) as a function of time, determining the time interval $\tau$ between two successive maxima of this function.

In case of obtaining a sequence of attenuated echo-signals, the thickness of the testing object is calculated by the following formula: $h=0.5 \cdot \tau_{1, k} \cdot c /(k-1)$, where $\tau_{1, k}$ - the time interval between the first and $k$-th echo-signal. According to this, the root-mean-square value of the error of time interval quantization decreases by the factor of $\sqrt{k-1}$.

The plots of typical experimental data and calculated functions are shown in Fig.15. The frequency of the filling signal was $2.23 \mathrm{MHz}$.
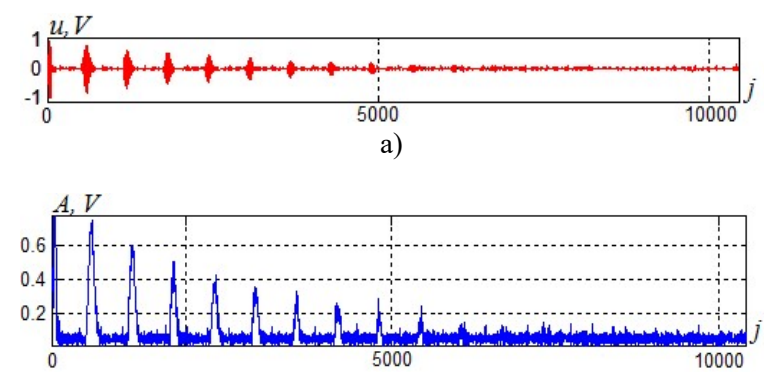

b)

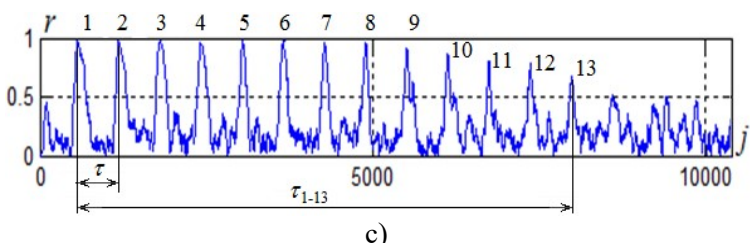

Fig.15. The plots of experimental results: a) - sequence $u[j]$;

b) - sequence $A[j] ; \mathrm{c})$ - sequence $r\left[j, M_{W}\right]$.

In this experiment, the echo signals on the noise background are difficult to distinguish using the signal envelope $A[j]=\sqrt{(u[j])^{2}+(\tilde{u}[j])^{2}}$ after the ninth pulse (see Fig.15.b)). However, the plot of the sequence $r\left[j, M_{W}\right]$ indicates that 13 echo-signals are easily detected in this experiment (see Fig.15.c)).

The considered applications do not exhaust all possible variants of use of the signal phase and its features in computerized systems of measurement and testing. Particularly in the field of surface metrology [45], [46].

\section{CONCLUSIONS}

The following conclusion can be made from this research.

The DHT-based method of phase measurements in conjunction with statistical processing of angular data makes it possible to more fully use the information hidden in the signal phase and to propose the use of several new features, which expands the information base of phase measurements. In particular, it allows: 
- simultaneously determining the phase, frequency and amplitude of signals, which is important for many technical applications;

- carrying out the phase analysis of signal when a phase difference is a slowly changing time function;

- obtaining samples of phase values, phase differences, amplitudes and frequencies of significant volumes and applying the statistical processing methods to them, which is important for the analysis of signals under noise.

The advantages of the DHT for phase measurements, as well as probabilistic space and model of random phase differences, are given. The possibility of using the theoretical basis of directional statistics data in phase measurements is justified.

Presented examples of using signal phase applied in measurements and NDT demonstrate the increasing of the uncertainty 5 times during measuring the diameter and increasing probability of determining a crack by $10-15 \%$. Also, the proposed method of evaluation of a phase difference improves the uncertainty 4-5 times.

Future work will encompass both the issues of computer modeling of the different algorithms in processing circular data and the use of the derived statistical features of a signal phase difference in the various physical experiments. In particular, in the field of surface metrology [42], [43].

\section{REFERENCES}

[1] Kuts, Y., Kochan, O., Lysenko, I., Huminilovych, R. (2021). Methodology for measuring phase shifts of signals using discrete Hilbert transform. In 13th International Conference on Measurement. IEEE, 1821. https://doi.org/10.23919/Measurement52780.2021. 9446811.

[2] Witkovsky, V., Frollo, I. (2020). Measurement science is the science of sciences-there is no science without measurement. Measurement Science Review, 20 (1), 15. https://doi.org/10.2478/msr-2020-0001.

[3] Jun, S., Przystupa, K., Beshley, M., Kochan, O., Beshley, H., Klymash, M., Wang, J., Pieniak, D. (2020). A cost-efficient software based router and traffic generator for simulation and testing of IP network. Electronics, 9 (1), 40. https://doi.org/10.3390/electronics9010040.

[4] Michalowska, J., Tofil, A., Józwik, J., Pytka, J., Legutko, S., Siemiatkowski, Z., Lukaszewicz, A. (2019). Monitoring the risk of the electric component imposed on a pilot during light aircraft operations in a high-frequency electromagnetic field. Sensors, 19 (24), 5537. https://doi.org/10.3390/s19245537.

[5] Wang, J., Kochan, O., Przystupa, K., Su, J. (2019). Information-measuring system to study the thermocouple with controlled temperature field. Measurement Science Review, 19 (4), 161-169. https://doi.org/10.2478/msr-2019-0022.
[6] Macek, W. (2021). Correlation between fractal dimension and areal surface parameters for fracture analysis after bending-torsion fatigue. Metals, 11 (11), 1790. https://doi.org/10.3390/met11111790.

[7] Machin, G., Bojkovski, J., del Campo, D., Dogan, A.K., Fischer, J., Hermier, Y., Merlone, A., Nielsen, J., Peruzzi, A., Ranostaj, J., Strnad, R. (2014). A European roadmap for thermometry. International Journal of Thermophysics, 35 (3-4), 385-394. https://doi.org/10.1007/s10765-013-1554-4.

[8] Filtz, J.R., Wu, J., Stacey, C., Hollandt, J., Monte, C., Hay, B., Hameury, J., Villamanan, M.A., ThurzoAndras, E., Sarge, S. (2015). A European roadmap for thermophysical properties metrology. International Journal of Thermophysics, 36 (2-3), 516-528. https://doi.org/10.1007/s10765-014-1807-x.

[9] Glowacz, A. (2021). Ventilation diagnosis of angle grinder using thermal imaging. Sensors, 21 (8), 2853. https://doi.org/10.3390/+s21082853.

[10] Shu, C., Kochan, O. (2013). Method of thermocouples self verification on operation place. Sensors \& Transducers, 160 (12), 55-61.

[11] Jun, S., Kochan, O., Kochan, R. (2016). Thermocouples with built-in self-testing. International Journal of Thermophysics, 37 (4), 37. https://doi.org/10.1007/s10765-016-2044-2.

[12] Schnaid, F. (2009). In Situ Testing in Geomechanics: The Main Tests. CRC Press, ISBN 9780429152603. https://doi.org/10.1201/9781482266054.

[13] Chen, Y., Yang, J., Xu, Y., Jiang, S., Liu, X., Wang, Q. (2016). Status self-validation of sensor arrays using gray forecasting model and bootstrap method. IEEE Transactions on Instrumentation and Measurement, 65 (7), 1626-1640. doi.org/10.1109/TIM.2016.2540942.

[14] Guzela, S., Dzianik, F. (2020). The recuperative heat exchangers - the mean temperature difference in the special cases of heat transfer. Journal of Mechanical Engineering (Strojnicky časopis), 70 (1), 47-56. http://dx.doi.org/10.2478/scjme-2020-0005.

[15] Yeromenko, V., Kochan, O. (2013). The conditional least squares method for thermocouples error modeling. In 2013 IEEE 7th International Conference on Intelligent Data Acquisition and Advanced Computing Systems (IDAACS). IEEE, Vol. 1, 157-162. https://doi.org/10.1109/IDAACS.2013.6662661.

[16] Mitryasova, O., Pohrebennyk, V., Cygnar, M., Sopilnyak, I. (2016). Environmental natural water quality assessment by method of correlation analysis. In 16th International Multidisciplinary Scientific GeoConference (SGEM 2016), 317-324. https://doi.org/10.5593/SGEM2016/B52/S20.041. 
[17] $\mathrm{Hu}, \mathrm{Z} ., \mathrm{Su}$, J., Jotsov, V., Kochan, O., Mykyichuk, M., Kochan, R., Sasiuk, T. (2016). Data science applications to improve accuracy of thermocouples. In 2016 IEEE 8th International Conference on Intelligent Systems (IS). IEEE, 180-188.

https://doi.org/10.1109/IS.2016.7737419.

[18] Glowacz, A. (2021). Fault diagnosis of electric impact drills using thermal imaging. Measurement, 171, 108815. https://doi.org/10.1016/j.measurement.2020.108815.

[19] Ignatiev, V., Nikitin, A., Yushanov, S. (2013). Measurement of phase shifts of quasiharmonic signals. Numerical Methods and Programming, 14 (4), 424431. (in Russian)

[20] Kuts, Y., Shcherbak, L. (2009). Statistical Phase Measurement. Ternopil, Ukraine: Ternopil Ivan Puluj National Technical University Press. (in Ukrainian)

[21] Dorozhovets, M., Motalo, V., Stadnyk, B., Vasyliuk, V., Borek, R., Kovalchyk, A. (2005). Fundamentals of Metrology and Measuring Techniques, Volume 2. Lviv, Ukraine: Lviv Polytechnic National University Press. (in Ukrainian)

[22] Tu, Y., Yang, H., Zhang, H., Liu, X. (2014). CMF signal processing method based on feedback corrected ANF and Hilbert transformation. Measurement Science Review, 14 (1), 41-47. https://doi.org/10.2478/msr-2014-0007.

[23] Liu, C.Y., Wang, C.Y. (2020). Investigation of phase pattern modulation for digital fringe projection profilometry. Measurement Science Review, 20 (1), 43-49. https://doi.org/10.2478/msr-2020-0006.

[24] Sedlacek, M., Krumpholc, M. (2005). Digital measurement of phase difference - a comparative study DSP algorithms. Metrology and Measurement Systems, 12 (4), 427-448.

[25] Wang, K., Tu, Y., Shen, Y., Xiao, W., McLernon, D. (2018). A modulation based phase difference estimator for real sinusoids to compensate for incoherent sampling. Review of Scientific Instruments, 89 (8), 085120. https://doi.org/10.1063/1.5026439.

[26] Shen, Y.L., Tu, Y.Q., Chen, L.J., Shen, T.A. (2015). Phase difference estimation method based on data extension and Hilbert transform. Measurement Science and Technology, 26 (9), 095003.

https://doi.org/10.1088/0957-0233/26/9/095003.

[27] Ignatjev, V., Stankevich, D. (2017). A fast estimation method for the phase difference between two quasiharmonic signals for real-time systems. Circuits, Systems, and Signal Processing, 36 (9), 3854-3863. https://doi.org/10.1007/s00034-016-0484-3.

[28] Chen, N., Fan, S., Zheng, D. (2019). A phase difference measurement method based on strong tracking filter for Coriolis mass flowmeter. Review of Scientific Instruments, 90 (7), 075003. https://doi.org/10.1063/1.5086714.
[29] Zhang, M., Wang, H., Qin, H., Zhao, W., Liu, Y. (2018). Phase difference measurement method based on progressive phase shift. Electronics, 7 (6), 86. https://doi.org/10.3390/electronics7060086.

[30] Choi, U.G., Kim, H.Y., Han, S.T., Yang, J.R. (2019). Measurement method of amplitude ratios and phase differences based on power detection among multiple ports. IEEE Transactions on Instrumentation and Measurement, 68 (12), 4615-4617. https://doi.org/10.1109/TIM.2019.2943976.

[31] Gula, V., Polikarovskykh, O., Horiashchenko, K., Karpova, L.V., Melnychuk, V.M. (2019). Measurements of periodic signals phase shifts with application of direct digital synthesis. Devices and Methods of Measurements, 10 (2), 169-177.

https://doi.org/10.21122/2220-9506-2019-10-2-169177.

[32] Joint Committee for Guides in Metrology. (2012). International vocabulary of metrology - Basic and general concepts and associated terms (VIM), 3rd edition. JCGM 200:2012.

[33] Babak, V., Babak, S., Eremenko, V., Kuts, Y., Myslovych, M., Scherbak, L., Zaporozhets, A. (2021). Models and Measures in Measurements and Monitoring. Springer, ISBN 978-3-030-70782-8. https://doi.org/10.1007/978-3-030-70783-5.

[34] Bendat, J., Piersol, A. (2010). Random Data. Analysis and Measurement Procedures. John Willey \& Sons. ISBN 978-0-470-24877-5.

[35] Poularikas, A. (2010). Transforms and Applications Handbook. CRC Press. ISBN 9781420066524.

[36] Mardia, K.V., Jupp, P.E. (1999). Directional Statistics. John Willey \& Sons. ISBN 978-0-471-95333-3.

[37] Fisher, N. (2011). Statistical Analysis of Circular Data. Cambridge University Press. https://doi.org/10.1017/CBO9780511564345.

[38] Marple, L. (1999). Computing the discrete-time "analytic" signal via FFT. IEEE Transactions on Signal Processing, 47 (9), 2600-2603. https://doi.org/10.1109/78.782222.

[39] Kuts, Y., Protasov, A., Lysenko, I., Dugin, O., Bliznuk, O., Uchanin, V. (2017). Using multidifferential transducer for pulsed eddy current object inspection. In 2017 IEEE First Ukraine Conference on Electrical and Computer Engineering (UKRCON). IEEE, 826-829. https://doi.org/10.1109/UKRCON.2017.8100361.

[40] Lysenko, I., Protasov, A., Kuts, Y., Dugin, O. (2017). Pulsed eddy current non-destructive testing. Scientific Proceedings of STUME, 1 (216), 114-117.

[41] Kuts, Y., Protasov, A., Lysenko, I., Dugin, O. (2014). Pulsed eddy current non-destructive testing of the coating thickness. In 11th European Conference on Non-Destructive Testing (ECNDT 2014). Brno, Czech Republic: Brno University of Technology, ISBN 97880-214-5018-9, 1-8. 
[42] Uchanin, V.M. (2013). Overhead Eddy Current Converters of Double Differentiation. Lviv, Ukraine: SPLOM.

[43] Lysenko, I., Kuts, Y., Eremenko, V., Protasov, A., Uchanin, V. (2020). Advanced signal processing methods for inspection of aircraft structural materials. Transactions on Aerospace Research, 2 (259), 27-35. http://dx.doi.org/10.2478/tar-2020-0008.

[44] Kuts, Y., Monchenko, O., Bystra, I., et al. (2019). Phase method of ultrasonic pulse-echo thickness measurement of products made of structural materials. Interservise, Kyiv, Ukraine. (in Ukrainian)
[45] Macek, W., Marciniak, Z., Branco, R., Rozumek, D., Królczyk, G.M. (2021). A fractographic study exploring the fracture surface topography of S355J2 steel after pseudo-random bending-torsion fatigue tests. Measurement, 178, 109443. https://doi.org/10.1016/j.measurement.2021.109443.

[46] Maruda, R.W., Krolczyk, G.M., Wojciechowski, S., Powalka, B., Klos, S., Szczotkarz, N., Matuszak, M., Khanna, N. (2020). Evaluation of turning with different cooling-lubricating techniques in terms of surface integrity and tribologic properties. Tribology International, 148, 106334.

https://doi.org/10.1016/j.triboint.2020.106334.

Received November 09, 2021

Accepted January 13, 2022 